\title{
Clinical efficacy of intravenous amiodarone in the short term treatment of recurrent sustained ventricular tachycardia and ventricular fibrillation
}

\author{
WILHELM SCHÜTZENBERGER, FRANZ LEISCH, KLAUS KERSCHNER, \\ WOLFGANG HARRINGER, WALTER HERBINGER
}

From the 1 Medizinische Abteilung des Allgemeinen Krankenhauses der Stadt Linz, Austria

SUMMARY The clinical efficacy of intravenous amiodarone in terminating sustained ventricular tachycardia and in preventing recurrences of ventricular tachycardia and ventricular fibrillation was evaluated in 26 patients. All of them presented with organic heart disease accompanied by depressed left ventricular function. Intravenous amiodarone terminated spontaneous ventricular tachycardia in eight of 19 patients. Fifteen of the 26 patients had had at least one episode of ventricular tachycardia or ventricular fibrillation each day in the period immediately before the intravenous administration of amiodarone. Amiodarone controlled ventricular tachycardia or ventricular fibrillation in nine of these 15 patients; in three further cases it was successful when supplemented by additional administration of a previously ineffective antiarrhythmic drug and ventricular pacing. Two patients died despite these measures. In one, the amiodarone infusion had to be stopped because of an arrhythmogenic effect. Sustained deterioration of haemodynamic function or of pre-existing intraventricular conduction disturbances was never seen.

Intravenous amiodarone was effective in terminating sustained ventricular tachycardia and in preventing frequent episodes of ventricular arrhythmia that were refractory to other antiarrhythmic drugs.

Long term treatment with oral amiodarone is effective in ventricular tachycardia and ventricular fibrillation refractory to conventional antiarrhythmic drugs. ${ }^{1-3}$ Prolonged administration is required to achieve these antiarrhythmic effects, and oral amiodarone is not an appropriate treatment for acute tachycardia. ${ }^{4}$ Intravenous amiodarone, however, had a rapid effect on supraventricular tachycardias. ${ }^{56}$ Its role in the short term treatment of life threatening ventricular arrhythmias has not yet been determined. We report the results of using intravenous amiodarone for the termination and short term control of recurrent sustained ventricular tachycardia and ventricular fibrillation.

\section{Patients and methods}

We treated 26 patients (table 1) with recurrent

Requests for reprints to Dr Wilhelm Schützenberger, Allgemeines Krankenhaus der Stadt Linz, Krankenhausstrasse 9, 4020 Linz, Austria.

Accepted for publication 25 April 1989 sustained ventricular tachycardia or ventricular fibrillation with a standardised dose of intravenous amiodarone.

Table 2 lists the antiarrhythmic agents that failed to suppress tachycardia before intravenous amiodarone was tried. The following were administered: intravenous lignocaine (a loading dose of 1 to $2 \mathrm{mg} / \mathrm{kg}$ body weight followed by a continuous infusion of 2-4

Table 1 Clinical features of 26 patients treated with intravenous amiodarone

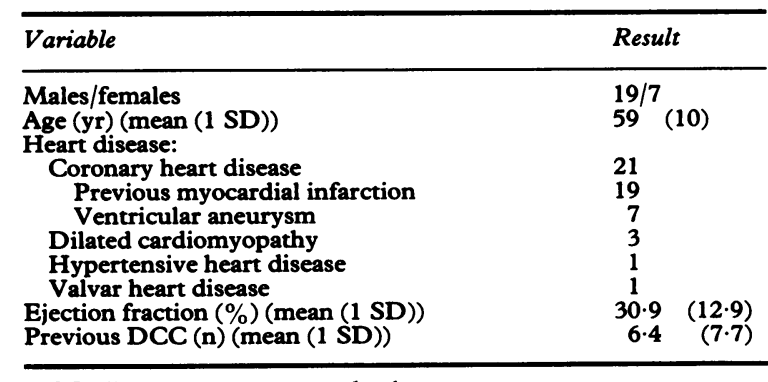

DCC, direct current countershock. 
Table 2 Unsuccessful drug treatment

\begin{tabular}{|c|c|c|}
\hline Case & Intravenous & Oral \\
\hline $\begin{array}{r}1 \\
2 \\
3 \\
4 \\
5 \\
6 \\
7 \\
8 \\
9 \\
10 \\
11 \\
12 \\
13 \\
14 \\
15 \\
16 \\
17 \\
18 \\
19 \\
20 \\
21 \\
22 \\
23 \\
24 \\
25 \\
26\end{array}$ & $\begin{array}{l}\text { L, P, M } \\
\text { L, P } \\
\text { L, P } \\
\text { L, M } \\
\text { L, M } \\
\text { L, P } \\
\text { L, P, M } \\
\text { L, P, M } \\
\text { L, P } \\
\text { L, P, M } \\
\text { L } \\
\text { L } \\
\text { L, P, M } \\
\text { L, P, M } \\
\text { L, P, M } \\
\text { L, P, M } \\
\text { L , P, M } \\
\text { L, P, M } \\
\text { L, P, M } \\
\text { L, P M } \\
\text { L, P, M } \\
\text { L, P }\end{array}$ & 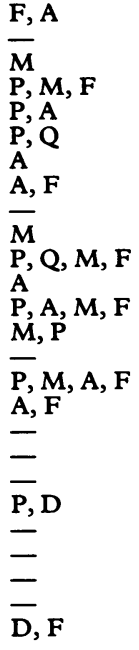 \\
\hline
\end{tabular}

A, prajmalium bitartrate; $D$, disopyramide; F, flecainide; L, lignocaine; $M$, mexiletine; $P$, propafenone; $Q$, quinidine.

$\mathrm{mg} / \mathrm{min}$ ); intravenous mexiletine (a loading dose of $100-250 \mathrm{mg}$, followed by $250 \mathrm{mg}$ for the next hour, another $250 \mathrm{mg}$ for the next two hours, and subsequently a continuous infusion of 1 to $2 \mathrm{mg} / \mathrm{min}$ ); intravenous propafenone (a loading dose of $1 \mathrm{mg} / \mathrm{kg}$ body weight followed by a continuous infusion of $0.5-1 \mathrm{mg} / \mathrm{min}$ ); oral prajmalium bitartrate (80 $\mathrm{mg} /$ day); oral disopyramide (400 $\mathrm{mg} /$ day); oral flecainide (200-300 mg/day); oral mexiletine (a loading dose of $1000 \mathrm{mg}$ in the first 24 hours followed by $600 \mathrm{mg} /$ day); oral propafenone $(900 \mathrm{mg} /$ day); oral quinidine $1.2 \mathrm{~g} /$ day. Amiodarone was given as follows: $5 \mathrm{mg} / \mathrm{kg}$ body weight in the form of a short term infusion within 20 minutes, followed by a continuous intravenous infusion of $1050 \mathrm{mg}$ over 24 hours. Oral treatment was started on the second day with $600-800 \mathrm{mg} /$ day for the next 13 days, followed by a maintenance dose of $400 \mathrm{mg} /$ day. The electrocardiogram and blood pressure were monitored continuously in the coronary care unit. The last electrocardiogram recorded before the administration of intravenous amiodarone and the one immediately after the conclusion of the 24 hour amiodarone infusion were used for the electrocardiographic diagnosis.

Serum concentrations of amiodarone and desethyl amiodarone were measured by high pressure liquid chromatography.

The results were analysed for statistical significance by Student's $t$ test.

\section{Results}

\section{SHORT TERM CONVERSION OF SUSTAINED}

VENTRICULAR TACHYCARDIA TO SINUS RHYTHM

We attempted to terminate a ventricular tachycardia with intravenous amiodarone in 19 of our 26 patients. Termination of ventricular tachycardia was not seen in any of them without medical or electrical measures. All of these patients were haemodynamically stable at the beginning of the infusion. Electrical cardioversion was performed whenever the ventricular tachycardia was not terminated $60 \mathrm{~min}-$ utes after the start of intravenous amiodarone or whenever the patient became haemodynamically unstable. The intravenous amiodarone was effective in terminating a spontaneous ventricular tachycardia in eight out of 19 patients within $31 \cdot 1$ (20) minutes. The termination was preceded by a decrease in the tachycardia cycle length in three patients, but occurred abruptly without prior reduction of cycle length in the remaining five. Comparison of the tachycardia rates shows that the mean value (161.9 (19.0)/min) was significantly lower in the group in which drug administration terminated the arrhythmia ( $\mathrm{p}<0.05)$ than in the patients who ultimately had to be treated with cardioversion (187.5 (24.7)/ min). The difference in ejection fractions between both groups (drug treatment $25 \cdot 8(8 \cdot 2) \%$, electrical cardioversion $31.7(9.6) \%$ ) was not statistically significant.

PREVENTION OF FREQUENT RECURRENCES OF VENTRICULAR TACHYCARDIA AND VENTRICULAR FIBRILLATION

Fifteen of the 26 patients had at least one episode of ventricular tachycardia or ventricular fibrillation every day in the period (3-8 days) immediately preceding the intravenous amiodarone treatment. Because of severe heart failure, three out of the 15 were being treated with dopamine or dobutamine before the amiodarone infusion. In nine of the 15 patients tachycardia was suppressed solely with intravenous amiodarone. Six, however, continued to have episodes of sustained ventricular tachycardia or ventricular fibrillation during the first 24 hours without change in cycle length. These could not be suppressed even with a further short term infusion. In three of the six patients short term control of the arrhythmia was achieved by supplementing the treatment with a previously ineffective antiarrhythmic drug and ventricular pacing. Despite these measures two patients continued to have frequent episodes of the same arrhythmia they showed before treatment and they died of an arrhythmic cause. In the sixth patient, the amiodarone infusion had to be stopped because of an increase in ventricular tachycardia with 
transition to ventricular fibrillation. During the second and third week three patients had repeated recurrences of spontaneous clinical ventricular tachycardia on oral amiodarone treatment despite combination with other antiarrhythmic agents. Catheter electrode ablation was attempted in one. This proved unsuccessful, however, and an antitachycardia pacemaker had to be implanted. Coronary bypass surgery and an aneurysmectomy were performed in the other two and in the patient who showed an arrhythmogenic effect in response to amiodarone.

The following side effects were seen: thrombophlebitis on seven occasions, flush once, sinus arrest after electrical cardioversion once, and an increase of arrhythmia once. In the patient with the sinus arrest a normal sinus rhythm was restored immediately after the intravenous administration of $1 \mathrm{mg}$ atropine. Hypotension, which we saw five times during short term infusion, and persistent ventricular tachycardia were rapidly eliminated by cardioversion.

\section{ELECTROCARDIOGRAPHIC FINDINGS}

Table 3 shows that the RR and PR intervals were not affected by intravenous amiodarone. Neither was the QTc time nor the QRS duration. Eleven of the 26 patients had conduction disorders before the intravenous administration of amiodarone: first degree atrioventricular block in three cases, right bundle branch block in two, and a left bundle branch block in two. Two further patients had an incomplete right bundle branch block, and two an incomplete left bundle branch block. Intravenous amiodarone, however, never caused a deterioration in atrioventricular or intraventricular conduction in any of the 11 patients with pre-existing conduction disorders, neither did it provoke new disturbances of this type in any of the remaining 15 patients.

\section{SERUM CONCENTRATIONS OF AMIODARONE AND DESETHYL AMIODARONE CONCENTRATION (figure 1)}

In three patients the serum concentrations of amiodarone and desethyl amiodarone were measured immediately before and after the short term infusion. They were also measured every two hours during the

Table 3 Electrocardiographic effects of the amiodarone infusion (mean (1SD))

\begin{tabular}{lrrrr}
\hline Interval & \multicolumn{2}{c}{ Control (ms) } & \multicolumn{3}{c}{ Intravenous amiodarone (ms) } \\
\hline RR & 741 & $(206)$ & 713 & $(178)$ \\
PR & 180 & $(40)$ & 185 & $(33)$ \\
QRS & 99 & $(16)$ & 102 & $(1)$ \\
QTc & 462 & $(65)$ & 467 & $(53)$
\end{tabular}

There were no significant differences between the control and amiodarone results.

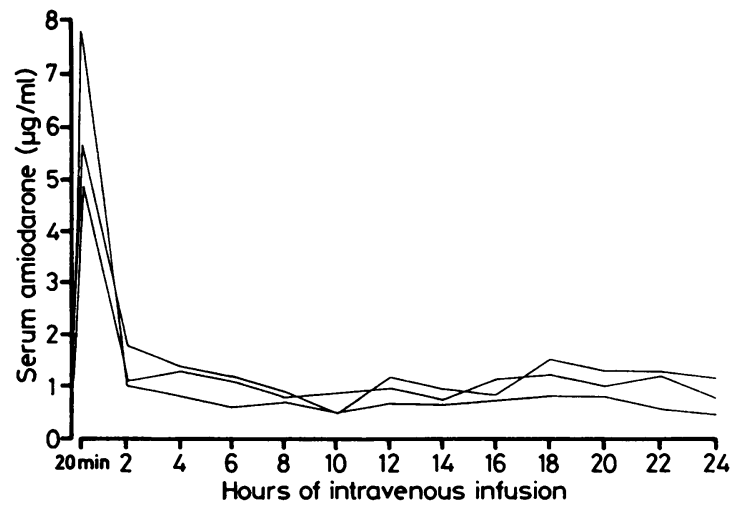

Figure Time course of serum amiodarone concentration during intravenous amiodarone infusion in three patients. Note the peak immediately after the short term infusion and the nearly constant blood concentrations from the second to the twenty fourth hour.

24 hour intravenous treatment. Serum concentrations of amiodarone reached an initial peak of between 4.9 and $7.8 \mu \mathrm{g} / \mathrm{ml}$ immediately after the short term infusion. During the succeeding period of observation they ranged from 0.5 to $1.5 \mathrm{~g} / \mathrm{ml}$. Concentrations of desethyl amiodarone, however, behaved differently. In one patient this metabolite was found four hours after treatment started, but in the remaining two it was never detected during the intravenous infusion.

\section{Discussion}

This study shows that intravenous amiodarone can be used safely and effectively for the short term control of frequent recurrent ventricular tachycardia and ventricular fibrillation and also for the termination of sustained ventricular tachycardia. The rate of termination in our patients-eight out of 19-is lower than the average of $70 \%$ in other reports. ${ }^{7-9}$ This discrepancy is primarily the result of different rates of amiodarone administration. We started treatment with a short term infusion rather than a bolus, which occasionally results in severe complications. ${ }^{4}$ In five patients in whom persistent ventricular tachycardia was rapidly corrected by cardioversion, hypotension occurred during short term infusion of amiodarone. Therefore close supervision and continuous monitoring not only of the electrocardiogram but also of blood pressure are recommended during intravenous amiodarone administration.

Of greater clinical significance than the termination of sustained ventricular tachycardia is the prompt efficacy with which amiodarone prevented ventricular tachycardia and ventricular fibrillation in 
our patients. Thus intravenous amiodarone on its own controlled tachycardia in nine of the 15 patients with frequent attacks of ventricular tachycardia or ventricular fibrillation that were refractory to other antiarrhythmic drugs. In addition three further patients were stabilised by supplementary administration of a previously ineffective antiarrhythmic drug and ventricular pacing. Similar positive results were also reported by Morady et al, who treated their patients mainly by repeating a bolus, ${ }^{10}$ and by Saksena et al, who performed continuous infusions for 4-7 days. ${ }^{11}$ Blood concentrations of amiodarone at the dosage we chose tend to be at the lower end of the range generally regarded as therapeutically effective. Therefore an increase of the dose infused over 24 hours might be expected to lead to better results, especially in patients with frequent attacks.

Only occasionally during the treatment with intravenous amiodarone did serious side effects occur that required the treatment to be stopped or the application of additional measures. The augmentation of the arrhythmias seen in one patient started 30 minutes after the start of treatment and persisted for about an hour after interruption of the infusion. This arrhythmogenic effect was not accompanied by a QTc prolongation, although such a correlation has been reported during oral amiodarone. ${ }^{12}{ }^{13}$ During the sinus arrest which occurred after cardioversion a supraventricular escape rhythm and a fall in blood pressure were seen. The immediate intravenous administration of $1 \mathrm{mg}$ atropine resulted in rapid restoration of sinus rhythm and normalisation of blood pressure. A few weeks before this patient showed normal sinus node function in a follow up electrophysiological study. Although a sinus arrest was reported to have been induced by intravenous amiodarone in one instance, the cause of the arrest in our case cannot be clearly attributed to the drug, because bradycardia seems to occur in $25 \%$ of all ventricular arrhythmias treated by cardioversion. ${ }^{14} 15$

All our patients showed depressed left ventricular function and some of them had severe congestive heart failure. Nevertheless we never saw a lasting deterioration of cardiac output. Others had confirmed that intravenous amiodarone is well tolerated provided bolus doses are avoided. ${ }^{10}{ }^{11}$ In our patients we found no alterations in the surface electrocardiogram during the 24 hour infusion of amiodarone. This observation was also reported by Wellens et al. ${ }^{16}$ But Saksena et al found a prolongation of the RR, PR, QRS, and QTc intervals. ${ }^{11}$ Because their infusion lasted 4-7 days, these changes are likely to be typical type III effects like those produced by long term oral administration. In long term oral treatment there is a correlation between antiarrhythmic action and QTc prolongation. ${ }^{17}{ }^{18}$ Because a QTc prolonga- tion cannot be detected during short term intravenous amiodarone administration, the short term effect in intravenous administration seems to be based upon the inhibition of the rapid inward sodium current, which was shown especially during acute superfusion with the drug in previously untreated papillary muscles by Mason et al. ${ }^{19}$ An effect of amiodarone on the rapid inward sodium current was also suggested earlier by Coumel and Bouvrain ${ }^{20}$ and Surawicz. ${ }^{21}$

Neither the short term infusion nor the subsequent 24 hour intravenous administration of amiodarone led to a deterioration of the atrioventricular or intraventricular conduction in any of our patients. This also held true when there was a pre-existing conduction disorder. Because of the effect of the drug on the atrioventricular node it should only be given after insertion of a pacemaker lead in patients with atrioventricular block.

\section{LIMITATIONS OF THE STUDY}

Because amiodarone was administered intravenously in all 26 patients we are not able to make a statement about the efficacy of this drug compared with that of other intravenously administered antiarrhythmics. A controlled comparative study, however, is impracticable because it would not be acceptable to stop a successful treatment in critically ill patients. Because all 26 patients were treated with other antiarrhythmic agents until just before the start of intravenous treatment, an additive effect cannot be ruled out. None the less, these antiarrhythmic agents were not effective when administered alone.

Intravenous amiodarone treatment with the specified dosage is effective both in the termination of sustained ventricular tachycardia and in the acute control of frequently recurring ventricular tachycardia and ventricular fibrillation that are refractory to treatment with other antiarrhythmic agents. With appropriate monitoring intravenous amiodarone can be used safely even in patients with left ventricular dysfunction and intraventricular conduction disturbances.

\section{References}

1 Morady F, Sauve MJ, Malone P, et al. Long-term efficacy and toxicity of high-dose amiodarone therapy for ventricular tachycardia or ventricular fibrillation. Am J Cardiol 1983;52:975-9.

2 McGovern B, Garan H, Malacoff RF, et al. Long-term clinical outcome of ventricular tachycardia or fibrillation treated with amiodarone. Am J Cardiol 1984;53: 1558-63.

3 Heger JJ, Prystowsky EN, Zipes DP. Clinical efficacy of amiodarone in treatment of recurrent ventricular tachycardia and ventricular fibrillation. Am Heart $J$ 
1983;106:887-94.

4 Marens FJ, Fontaine GH, Frank R, Grosgogeat Y. Clinical pharmacology and therapeutic applications of the antiarrhythmic agent amiodarone. Am Heart $J$ 1981;101:480-93.

5 Holt P, Crick JCP, Davies DW, Curry P. Intravenous amiodarone in the acute termination of supraventricular arrhythmias. Int J Cardiol 1985;8:67-76.

6 Faniel R, Schoenfeld P. Efficacy of i.v. amiodarone in converting rapid atrial fibrillation and flutter to sinus rhythm in intensive care patients. Eur Heart $J$ 1983; 4:180-5.

7 Benaim R, Uzan C. Les effets antiarhythmiques de l'amiodarone injectable (a propos de $153 \mathrm{cas}$ ). Rev Med 1978;19:1959-63.

8 Fauchier JP, Brochier M, Raynaud R. Etude clinique des effets antiarhythmiques ventriculaires de l'amiodarone (orale et injectable). Ann Cardiol Angeiol 1973;22:427-35.

9 Michat L, Cabrol C, Cabrol A, et al. Effets antiarhythmiques de l'amiodarone injectable en reanimation de chirurgie cardiovasculaire [Letter]. Nouv Presse Med 1976;5:1996.

10 Morady F, Schienman MM, Shen E, Shapiro W, Sung $\mathrm{RJ}$, Di Carlo L. Intravenous amiodarone in the acute treatment of recurrent symptomatic ventricular tachycardia. Am J Cardiol 1983;51:156-9.

11 Saksena S, Rothbart ST, Shah Y, Capello G. Clinical efficacy and electropharmacology of continuous intravenous amiodarone and chronic oral amiodarone in refractory ventricular tachycardia. Am J Cardiol 1984;54:347-52.

12 McGovern B, Garan H, Kelly E, Ruskin F. Adverse reactions during treatment with amiodarone hydrochloride. Br Med J 1983;287:175-80.

13 Leory G, Haiat R, Barthelemy M, Lionnet F. Torsade de pointes during loading with amiodarone. Eur Heart $J$ 1987;8:541-3.

14 Veltri EP, Reid $\mathrm{Ph} \mathrm{R}$. Sinus arrest with intravenous amiodarone. Am J Cardiol 1986;58:1110-1.

15 Eysmann SB, Marchlinski FE, Buxton AE, Josephson ME. Electrocardiographic changes after cardioversion of ventricular arrhythmias. Circulation 1986; 73:73-81.

16 Wellens HJJ, Brugada P, Abdollah H, Dassen WR. A comparison of the electrophysiologic effects of intravenous and oral amiodarone in the same patient. Circulation 1984;69:120-4.

17 Nademanee K, Hendrickson JA, Cannon OS, Goldreyer BN, Singh BN. Control of life-threatening ventricular tachyarrhythmias by amiodarone. $\mathrm{Am}$ Heart J 1981;101:759-68.

18 Singh BN, Nademanee K, Kannan R, Ikeda N. The clinical results of amiodarone in cardiac arrhythmias: optimal dosing. PACE 1984;7:109-24.

19 Mason JW, Hondeghem LM, Katzung BG. Block of inactivated sodium channels and of depolarizationinduced automatically in guinea pig papillary muscle by amiodarone. Circ Res 1984;55:277-85.

20 Coumel P, Bouvrain Y. Etude clinique des effets pharmacodynamiques et antiarythmiques de l'amiodarone. J Agrégés 1972;6:69-81.

21 Surawicz $B$. What protects the heart from standstill and fibrillation? In: Russek HI, ed. The Paul D. White symposium on cardiovascular disease. Baltimore: Williams and Wilkins, 1973:320-34. 